\title{
Boundary Value Problems for Fourth Order Nonlinear $p$-Laplacian Difference Equations
}

\author{
Qinqin Zhang ${ }^{1,2}$ \\ ${ }^{1}$ School of Mathematics and Information Science, Guangzhou University, Guangzhou, Guangdong 510006, China \\ ${ }^{2}$ Key Laboratory of Mathematics and Interdisciplinary Sciences of Guangdong Higher Education Institutes, \\ Guangzhou University, Guangzhou, Guangdong 510006, China
}

Correspondence should be addressed to Qinqin Zhang; qinqin.zhang0413@gmail.com

Received 6 October 2013; Accepted 26 December 2013; Published 30 January 2014

Academic Editor: Shih-sen Chang

Copyright (c) 2014 Qinqin Zhang. This is an open access article distributed under the Creative Commons Attribution License, which permits unrestricted use, distribution, and reproduction in any medium, provided the original work is properly cited.

We consider the boundary value problem for a fourth order nonlinear $p$-Laplacian difference equation containing both advance and retardation. By using Mountain pass lemma and some established inequalities, sufficient conditions of the existence of solutions of the boundary value problem are obtained. And an illustrative example is given in the last part of the paper.

\section{Introduction}

Let $\mathbb{N}, \mathbb{Z}$, and $\mathbb{R}$ denote the sets of all natural numbers, integers, and real numbers, respectively. For $a, b \in \mathbb{Z}$, define $\mathbb{Z}(a)=\{a, a+1, \ldots\}$ and $\mathbb{Z}(a, b)=\{a, a+1, \ldots, b\}$ when $a \leq b$.

Consider the following fourth order nonlinear difference equation:

$$
\begin{array}{r}
\Delta^{2}\left(r_{n-2} \phi_{p}\left(\Delta^{2} u_{n-2}\right)\right)-f\left(n, u_{n+1}, u_{n}, u_{n-1}\right)=0, \\
n \in \mathbb{Z}(1, k),
\end{array}
$$

with boundary value conditions

$$
u_{-1}=u_{0}=u_{k+1}=u_{k+2}=0,
$$

where $k \in \mathbb{N}, r_{j}$ is a positive number for $j \in \mathbb{Z}(-1, k), \Delta$ is the forward difference operator defined by $\Delta u_{n}=u_{n+1}-u_{n}$, $\Delta^{2} u_{n}=\Delta\left(\Delta u_{n}\right)$, and $\phi_{p}$ is the $p$-Laplacian operator; that is, $\phi_{p}(s)=|s|^{p-2} s(p>1), f \in C\left(\mathbb{Z}(1, k) \times \mathbb{R}^{3}, \mathbb{R}\right)$.

In the last decade, by using various techniques such as critical point theory, fix point theory, topological degree theory, and coincidence degree theory, a great deal of works have been done on the existence of solutions to boundary value problems of difference equations (see [1-7] and references therein). Among these approaches, the critical point theory seems to be a powerful tool to deal with this problem (see [5, 7-9]). However, compared to the boundary value problems of lower order difference equations $([6,8$, $10-13])$, the study of boundary value problems of higher order difference equations is relatively rare (see $[9,14,15])$, especially the works by using the critical point theory [16]. For the background on difference equations, we refer to [17].

In this paper, we will consider the existence of solutions of the boundary value problem of (1) with (2). First, we will construct a functional $J$ such that solutions of the boundary value problem (1) with (2) correspond to critical points of $J$. Then, by using Mountain pass lemma, we obtain the existence of critical points of $J$. We mention that (1) is a kind of difference equation containing both advance and retardation. This kind of difference equation has many applications both in theory and practice. For example, in [17], Agarwal considered the following difference equation:

$$
-\omega^{2} M u(k)+f(-u(k-1)+2 u(k)-u(k+1))=0,
$$

with the boundary value conditions

$$
u(0)=u(k+1)=0,
$$

as an example. It represents the amplitude of the motion of every particle in the string. And in [7], the authors considered the following second order functional difference equation:

$$
L u_{n}=f\left(n, u_{n+1}, u_{n}, u_{n-1}\right),
$$


with different boundary value conditions

$$
\Delta x_{0}=A, \quad x_{T+1}=B,
$$

where the operator $L$ is the Jacobi operator given by

$$
L u_{n}=a_{n} u_{n+1}+a_{n-1} u_{n-1}+b_{n} u_{n}
$$

In [18], the authors considered the second order $p$-Laplacian difference equation:

$$
\Delta\left(\phi_{p}\left(\Delta u_{n-1}\right)\right)+f\left(n, u_{n+1}, u_{n}, u_{n-1}\right)=0, \quad n \in \mathbb{Z}(1, k),
$$

with boundary value conditions

$$
u_{0}=u_{k+1}=0
$$

As for the periodic and subharmonic solutions of $p$-Laplacian difference equations containing both advance and retardation, we refer to [19]. And for the periodic solutions of $\phi$ Laplacian difference equations, we refer to [20].

Throughout this paper, we assume that there exists a function $F(n, u, v)$ which is differentiable in $(u, v)$ and $F(n, 0,0)=$ 0 for each $n \in \mathbb{Z}(0, k)$, satisfying

$$
\begin{array}{r}
\frac{\partial F(n-1, v, w)}{\partial v}+\frac{\partial F(n, u, v)}{\partial v}=f(n, u, v, w), \\
\forall u, v, w \in \mathbb{R},
\end{array}
$$

for $n \in \mathbb{Z}(1, k)$.

\section{Preliminaries and Main Results}

Lemma 1. Let $p \in(1, \infty)$; then there exist two positive sequences $\left\{c_{*}(n)\right\}_{n \in \mathbb{N}}$ and $\left\{c^{*}(n)\right\}_{n \in \mathbb{N}}$ such that

$$
\begin{aligned}
c_{*}(b-a+1)\left(\sum_{j=a}^{b}\left|u_{j}\right|^{2}\right)^{1 / 2} & \leq\left(\sum_{j=a}^{b}\left|u_{j}\right|^{p}\right)^{1 / p} \\
& \leq c^{*}(b-a+1)\left(\sum_{j=a}^{b}\left|u_{j}\right|^{2}\right)^{1 / 2}
\end{aligned}
$$

holds for any $a, b \in \mathbb{Z}$ with $a<b$, where $c_{*}(n)=1, c^{*}(n)=$ $n^{(2-p) / 2 p}$ for $p \in(1,2]$ and $c_{*}(n)=n^{-(p-2) / 2 p}, c^{*}(n)=1$ for $p \in(2, \infty)$.

Proof. If $1<p \leq 2$, by Hölder's inequality, we have

$$
\begin{aligned}
\sum_{j=a}^{b}\left|u_{j}\right|^{p} & \leq\left(\sum_{j=a}^{b} 1^{2 /(2-p)}\right)^{(2-p) / 2}\left(\sum_{j=a}^{b}\left|u_{j}\right|^{2}\right)^{p / 2} \\
& =(b-a+1)^{(2-p) / 2}\left(\sum_{j=a}^{b}\left|u_{j}\right|^{2}\right)^{p / 2},
\end{aligned}
$$

which implies that

$$
\left(\sum_{j=a}^{b}\left|u_{j}\right|^{p}\right)^{1 / p} \leq(b-a+1)^{(2-p) / 2 p}\left(\sum_{j=a}^{b}\left|u_{j}\right|^{2}\right)^{1 / 2},
$$

and $c_{*}(n)=1$ is obvious. If $p>2$, then we have

$$
\begin{aligned}
\sum_{j=a}^{b}\left|u_{j}\right|^{2} & \leq\left(\sum_{j=a}^{b} 1^{p /(p-2)}\right)^{(p-2) / p}\left(\sum_{j=a}^{b}\left|u_{j}\right|^{p}\right)^{2 / p} \\
& =(b-a+1)^{(p-2) / p}\left(\sum_{j=a}^{b}\left|u_{j}\right|^{p}\right)^{2 / p},
\end{aligned}
$$

which implies that

$$
\left(\sum_{j=a}^{b}\left|u_{j}\right|^{p}\right)^{1 / p} \geq(b-a+1)^{-(p-2) / 2 p}\left(\sum_{j=a}^{b}\left|u_{j}\right|^{2}\right)^{1 / 2}
$$

and $c^{*}(n)=1$ is obvious. Now the proof is complete.

Lemma 2. There exist two positive sequences $\left\{\lambda_{*}(n)\right\}_{n \in \mathbb{N}}$ and $\left\{\lambda^{*}(n)\right\}_{n \in \mathbb{N}}$ such that

$$
\begin{aligned}
\lambda_{*}(b-a+1) \sum_{j=a}^{b} u_{j}^{2} & \leq \sum_{j=a-1}^{b}\left(\Delta u_{j}\right)^{2} \\
& \leq \lambda^{*}(b-a+1) \sum_{j=a}^{b} u_{j}^{2}
\end{aligned}
$$

holds for any $a, b \in \mathbb{Z}$ with $a<b$ and $u_{a-1}=u_{b+1}=0$, where

$$
\lambda_{*}(n)=4 \sin ^{2} \frac{\pi}{2(n+1)}, \quad \lambda^{*}(n)=4 \sin ^{2} \frac{n \pi}{2(n+1)} .
$$

Proof. There is no harm in assuming that $a=1, b=k$. Then

$$
\sum_{j=0}^{k}\left(\Delta u_{j}\right)^{2}=\left(u_{1}, u_{2}, \ldots, u_{k}\right) A\left(u_{1}, u_{2}, \ldots, u_{k}\right)^{\operatorname{tr}}
$$

where $(\cdot)^{\operatorname{tr}}$ means the transpose of $(\cdot)$, and $A$ is the $k \times k$ matrix given by

$$
A=\left(\begin{array}{cccccc}
2 & -1 & 0 & \cdots & 0 & 0 \\
-1 & 2 & -1 & \cdots & 0 & 0 \\
0 & -1 & 2 & \cdots & 0 & 0 \\
\cdots & \cdots & \cdots & \cdots & \cdots & \cdots \\
0 & 0 & 0 & \cdots & 2 & -1 \\
0 & 0 & 0 & \cdots & -1 & 2
\end{array}\right)
$$

We will calculate the eigenvalues of $A$. Similar to [21], assume that $\lambda$ is an eigenvalue of $A$. Since $A-r I$ is positive-definite for $r<0$ and negative-definite for $r>4$, where $I$ is the $k \times$ $k$ identity matrix, we see that $\lambda \in[0,4]$. Assume that $\xi=$ $\left(\xi_{1}, \xi_{2}, \ldots, \xi_{k}\right)^{\mathrm{tr}}$ is an eigenvector associated to $\lambda$ and define the sequence $\left\{y_{n}\right\}_{n=0}^{k+1}$ as

$$
y_{i}=\xi_{i}, \quad i \in \mathbb{Z}(1, k), \quad y_{0}=y_{k+1}=0 .
$$


Then $\left\{y_{n}\right\}$ satisfies

$$
\begin{array}{r}
-y_{n+1}+(2-\lambda) y_{n}-y_{n-1}=0, \quad y_{0}=y_{k+1}=0, \\
n \in \mathbb{Z}(1, k) .
\end{array}
$$

Since the roots of the equation $-r^{2}+(2-\lambda) r-1=0$ are

$$
\begin{aligned}
& r_{1}=\frac{1}{2}\left(2-\lambda+\sqrt{4-(2-\lambda)^{2}}\right) i \\
& r_{2}=\frac{1}{2}\left(2-\lambda-\sqrt{4-(2-\lambda)^{2}}\right) i
\end{aligned}
$$

set

$$
\theta=\arccos \frac{1}{2}(2-\lambda)
$$

Then

$$
y_{n}=d_{1} \cos n \theta+d_{2} \sin n \theta
$$

for some constants $d_{1}$ and $d_{2} \cdot y_{0}=0$ implies that $d_{1}=0$, and $y_{k+1}=0$ implies that $\sin (k+1) \theta=0$. Therefore, $(k+1) \theta=j \pi$ for $j \in \mathbb{Z}(1, k)$. By $(23)$, we have $\lambda=2(1-\cos \theta)=4 \sin ^{2} \theta / 2$ which implies that the eigenvalues of $A$ are

$$
\lambda_{j}=4 \sin ^{2} \frac{j \pi}{2(k+1)}, \quad j \in \mathbb{Z}(1, k) .
$$

The maximum eigenvalue of $A$ is $\lambda_{k}$, and the minimal eigenvalue of $A$ is $\lambda_{1}$. Equation (16) follows from (18) and the fact that $\lambda_{k}=\lambda^{*}(k)$ and $\lambda_{1}=\lambda_{*}(k)$.

Before we apply the critical point theory, we will establish the corresponding variational framework for (1) with (2).

Let

$$
\begin{aligned}
E=\{u & =\left\{u_{n}\right\}: \mathbb{Z}(-1, k+2) \\
& \left.\longrightarrow \mathbb{R} \mid u_{-1}=u_{0}=u_{k+1}=u_{k+2}=0\right\} .
\end{aligned}
$$

Then $E$ is a $k$-dimensional Hilbert space.

Obviously, $E$ is isomorphic to $\mathbb{R}^{k}$. In fact, we can find a map $I: E \rightarrow \mathbb{R}^{k}$ defined by

$$
I:\left\{u_{n}\right\} \longrightarrow\left(u_{1}, u_{2}, \ldots, u_{k}\right)^{\operatorname{tr}} .
$$

Define the inner product on $E$ as

$$
\langle u, v\rangle=\sum_{j=1}^{k} u_{j} v_{j}, \quad \forall u, v \in E
$$

The corresponding norm $\|\cdot\|$ can be induced by

$$
\|u\|=\left(\sum_{j=1}^{k}\left|u_{j}\right|^{2}\right)^{1 / 2}, \quad \forall u \in E .
$$

For all $u \in E$, define the functional $J(u)$ on $E$ as follows:

$$
J(u)=\frac{1}{p} \sum_{n=-1}^{k} r_{n}\left|\Delta^{2} u_{n}\right|^{p}-\sum_{n=0}^{k} F\left(n, u_{n+1}, u_{n}\right) .
$$

Clearly, $J \in C^{1}(E, \mathbb{R})$. We can compute the partial derivative as

$$
\frac{\partial J(u)}{\partial u_{j}}=\Delta^{2}\left(r_{j-2} \phi_{p}\left(\Delta^{2} u_{j-2}\right)\right)-f\left(j, u_{j+1}, u_{j}, u_{j-1}\right),
$$

for $j \in \mathbb{Z}(1, k), u=\left\{u_{j}\right\} \in E$. Therefore, $u \in E$ is a critical point of $J$ if and only if $u$ is a solution of (1) with (2).

Definition 3. Let $E$ be a real Banach space; the functional $J \in C^{1}(E, \mathbb{R})$ is said to satisfy the Palais-Smale (P.S. for short) condition if any sequence $\left\{x_{m}\right\}$ in $E$ such that $\left\{J\left(x_{m}\right)\right\}$ is bounded and $J^{\prime}\left(x_{m}\right) \rightarrow 0$ as $m \rightarrow \infty$ contains a convergent subsequence.

Let $B_{\rho}$ denote the open ball in $E$ with radius $\rho$ and center 0 , and let $\partial B_{\rho}$ denote its boundary.

In order to obtain the existence of critical points of $J$ on $E$, we need to use the following basic lemma, which is important in the proof of our main results.

Lemma 4 (Mountain pass lemma [22]). Let E be a real Hilbert space and $J \in C^{1}(E, \mathbb{R})$ satisfies the P.S. condition, if $J(0)=0$ and the following conditions hold.

$\left(J_{1}\right)$ There exist constants $a>0$ and $\rho>0$ such that $\left.J\right|_{\partial B_{\rho}} \geq$ a.

$\left(J_{2}\right)$ There exists $e \in E \backslash B_{\rho}$ such that $J(e)<0$.

Then J possesses a critical value $c \geq a$ given by

$$
c=\inf _{g \in \Gamma} \max _{s \in[0,1]} J(g(s)),
$$

where

$$
\Gamma=\{g \in C([0,1], E) \mid g(0)=0, g(1)=e\} .
$$

Let

$$
\begin{gathered}
r_{*}=\min \left\{r_{n} \mid n \in \mathbb{Z}(-1, k)\right\}, \\
r^{*}=\max \left\{r_{n} \mid n \in \mathbb{Z}(-1, k)\right\}, \\
\alpha_{0}=\frac{r_{*}}{p}\left(\frac{c_{*}(k+2)}{c^{*}(k+1)}\right)^{p}\left(\frac{\lambda_{*}(k+1) \lambda_{*}(k)}{2}\right)^{p / 2}, \\
\beta_{0}=\frac{r^{*}}{p}\left(\frac{c^{*}(k+2)}{c_{*}(k+1)}\right)^{p}\left(\frac{\lambda^{*}(k+1) \lambda^{*}(k)}{2}\right)^{p / 2} .
\end{gathered}
$$

Then, for $p \in(1,2]$,

$$
\begin{aligned}
& \alpha_{0}=\frac{r_{*}}{p}(k+1)^{-(2-p) / 2} 8^{p / 2}\left(\sin \frac{\pi}{2(k+2)} \sin \frac{\pi}{2(k+1)}\right)^{p}, \\
& \beta_{0}=\frac{r^{*}}{p}(k+2)^{(2-p) / 2} 8^{p / 2}\left(\sin \frac{(k+1) \pi}{2(k+2)} \sin \frac{k \pi}{2(k+1)}\right)^{p} .
\end{aligned}
$$


For $p \in(2, \infty)$,

$$
\begin{aligned}
& \alpha_{0}=\frac{r_{*}}{p}(k+2)^{-(p-2) / 2} 8^{p / 2}\left(\sin \frac{\pi}{2(k+2)} \sin \frac{\pi}{2(k+1)}\right)^{p}, \\
& \beta_{0}=\frac{r^{*}}{p}(k+1)^{(p-2) / 2} 8^{p / 2}\left(\sin \frac{(k+1) \pi}{2(k+2)} \sin \frac{k \pi}{2(k+1)}\right)^{p} .
\end{aligned}
$$

Now we state our main results.

Theorem 5. Assume that $F(n, u, v)$ satisfies the following conditions.

$\left(F_{1}\right)$ There exist constants $\delta \in(0, \infty)$ and $\alpha \in\left(0, \alpha_{0}\right)$ such that

$F(n, u, v) \leq \alpha\left(u^{2}+v^{2}\right)^{p / 2}, \quad$ for $n \in \mathbb{Z}(0, k)$

$$
u^{2}+v^{2} \leq \delta^{2}
$$

$\left(F_{2}\right)$ There exist constants $\beta \in\left(\beta_{0}, \infty\right)$ and $\gamma \in(0, \infty)$ such that

$F(n, u, v) \geq \beta\left(u^{2}+v^{2}\right)^{p / 2}-\gamma, \quad$ for $n \in \mathbb{Z}(0, k)$.

Then (1) with (2) possesses at least two nontrivial solutions.

Remark 6. Comparing our results with the results of the boundary value problems of second order $p$-Laplacian difference equations in [18], we find that our results are more precisely.

In view of (37) and (38), it is easy to obtain the following corollary.

Corollary 7. Assume that $F(n, u, v)$ satisfies

$$
\begin{gathered}
\lim _{u^{2}+v^{2} \rightarrow 0} \frac{F(n, u, v)}{\left(u^{2}+v^{2}\right)^{p / 2}}=0, \quad \forall n \in \mathbb{Z}(0, k), \\
\lim _{u^{2}+v^{2} \rightarrow+\infty} \frac{F(n, u, v)}{\left(u^{2}+v^{2}\right)^{p / 2}}=+\infty, \quad \forall n \in \mathbb{Z}(0, k) .
\end{gathered}
$$

Then (1) with (2) possesses at least two nontrivial solutions.

For the case when $p=2$, we have the following corollary for the boundary value problems of the fourth order nonlinear difference equations.

Corollary 8. Assume that $F(n, u, v)$ satisfies the following conditions.

$\left(F_{3}\right)$ There exist constants $\delta_{1}>0$ and $0<\alpha_{1}<\left(8 r_{*} / p\right)$ $(\sin (\pi / 2(k+2)) \sin (\pi / 2(k+1)))^{2}$ such that

$F(n, u, v) \leq \alpha_{1}\left(u^{2}+v^{2}\right), \quad$ for $n \in \mathbb{Z}(0, k)$,

$$
u^{2}+v^{2} \leq \delta_{1}^{2}
$$

$\left(F_{4}\right)$ There exist constants $\beta_{1}>\left(8 r^{*} / p\right)(\sin ((k+1) \pi / 2(k+$ 2)) $\sin (k \pi / 2(k+1)))^{2}$ and $\gamma_{1}>0$ such that

$$
F(n, u, v) \geq \beta_{1}\left(u^{2}+v^{2}\right)-\gamma_{1}, \quad \text { for } n \in \mathbb{Z}(0, k) \text {. }
$$

Then the following fourth order nonlinear difference equation

$$
\begin{array}{r}
\Delta^{2}\left(r_{n-2} \Delta^{2} u_{n-2}\right)-f\left(n, u_{n+1}, u_{n}, u_{n-1}\right)=0, \\
n \in \mathbb{Z}(1, k),
\end{array}
$$

with the boundary value conditions (2) possesses at least two nontrivial solutions.

\section{Proof of Theorem 5}

In order to prove Theorem 5, we first establish the following lemma.

Lemma 9. Assume that $F$ satisfies $\left(F_{2}\right)$; then the functional $J$ satisfies the P.S. condition.

Proof. Let $\left\{u^{(s)}\right\}_{s \in \mathbb{N}}$ be a sequence in $E$ such that $\left\{J\left(u^{(s)}\right)\right\}$ is bounded and $J^{\prime}\left(u^{(s)}\right) \rightarrow 0$ as $s \rightarrow \infty$. Then there exists a positive constant $C$ such that $\left|J\left(u^{(s)}\right)\right| \leq C$ for $s \in \mathbb{N}$.

By (11) and (16), we have

$$
\begin{aligned}
\frac{1}{p} \sum_{n=-1}^{k} r_{n}\left|\Delta^{2} u_{n}^{(s)}\right|^{p} \\
\leq \frac{r^{*}}{p}\left(c^{*}(k+2)\right)^{p}\left(\sum_{n=-1}^{k}\left|\Delta^{2} u_{n}^{(s)}\right|^{2}\right)^{p / 2} \\
\leq \frac{r^{*}}{p}\left(c^{*}(k+2)\right)^{p}\left(\lambda^{*}(k+1) \sum_{n=0}^{k}\left(\Delta u_{n}^{(s)}\right)^{2}\right)^{p / 2} \\
\leq \frac{r^{*}}{p}\left(c^{*}(k+2)\right)^{p}\left(\lambda^{*}(k+1) \lambda^{*}(k) \sum_{n=1}^{k}\left(u_{n}^{(s)}\right)^{2}\right)^{p / 2} \\
=\frac{r^{*}}{p}\left(c^{*}(k+2)\right)^{p}\left(\lambda^{*}(k+1) \lambda^{*}(k)\right)^{p / 2}\left\|u^{(s)}\right\|^{p} \\
\quad=\left(c_{*}(k+1)\right)^{p} 2^{p / 2} \beta_{0}\left\|u^{(s)}\right\|^{p} .
\end{aligned}
$$

And by $\left(F_{2}\right),(11)$, and (16), we have

$$
\begin{aligned}
\sum_{n=0}^{k} F\left(n, u_{n+1}^{(s)}, u_{n}^{(s)}\right) & \\
\geq & \beta \sum_{n=0}^{k}\left(\left(u_{n+1}^{(s)}\right)^{2}+\left(u_{n}^{(s)}\right)^{2}\right)^{p / 2}-(k+1) \gamma \\
\geq & \beta\left[c_{*}(k+1)\left(\sum_{n=0}^{k}\left(\left(u_{n+1}^{(s)}\right)^{2}+\left(u_{n}^{(s)}\right)^{2}\right)\right)^{1 / 2}\right]^{p} \\
& -(k+1) \gamma \\
= & \left(c_{*}(k+1)\right)^{p} 2^{p / 2} \beta\left\|u^{(s)}\right\|^{p}-(k+1) \gamma .
\end{aligned}
$$


Therefore, by (30), we obtain

$$
\begin{aligned}
J\left(u^{(s)}\right)= & \frac{1}{p} \sum_{n=-1}^{k} r_{n}\left|\Delta^{2} u_{n}^{(s)}\right|^{p}-\sum_{n=0}^{k} F\left(n, u_{n+1}^{(s)}, u_{n}^{(s)}\right) \\
\leq & \left(c_{*}(k+1)\right)^{p} 2^{p / 2}\left(\beta_{0}-\beta\right)\left\|u^{(s)}\right\|^{p} \\
& +(k+1) \gamma .
\end{aligned}
$$

Noticing that $J\left(u^{(s)}\right) \geq-C$ and $\beta>\beta_{0}$, by (45), we have

$$
\left\|u^{(s)}\right\|^{p} \leq \frac{(k+1) \gamma+C}{\left(c_{*}(k+1)\right)^{p} 2^{p / 2}\left(\beta-\beta_{0}\right)} .
$$

Since $E$ is a finite-dimensional space, (46) implies that $\left\{u^{(s)}\right\}$ is bounded and has a convergent subsequence. Thus P.S. condition is verified.

Now we give the proof of Theorem 5.

Proof. For any $u \in E$ with $\|u\| \leq \delta$, according to (11) and (16), we have

$$
\begin{aligned}
\frac{1}{p} \sum_{n=-1}^{k} r_{n}\left|\Delta^{2} u_{n}\right|^{p} & \\
\geq & \frac{r_{*}}{p}\left(c_{*}(k+2)\right)^{p}\left(\sum_{n=-1}^{k}\left|\Delta^{2} u_{n}\right|^{2}\right)^{p / 2} \\
\geq & \frac{r_{*}}{p}\left(c_{*}(k+2)\right)^{p}\left(\lambda_{*}(k+1) \sum_{n=0}^{k}\left(\Delta u_{n}\right)^{2}\right)^{p / 2} \\
\geq & \frac{r_{*}}{p}\left(c_{*}(k+2)\right)^{p}\left(\lambda_{*}(k+1) \lambda_{*}(k) \sum_{n=1}^{k}\left(u_{n}\right)^{2}\right)^{p / 2} \\
& =\frac{r_{*}}{p}\left(c_{*}(k+2)\right)^{p}\left(\lambda_{*}(k+1) \lambda_{*}(k)\right)^{p / 2}\|u\|^{p} .
\end{aligned}
$$

By $\left(F_{1}\right),(11)$, and $(16)$, we have

$$
\begin{aligned}
\sum_{n=0}^{k} F\left(n, u_{n+1}, u_{n}\right) & \\
\leq & \alpha \sum_{n=0}^{k}\left(u_{n+1}^{2}+u_{n}^{2}\right)^{p / 2} \\
& \leq \alpha\left[c^{*}(k+1)\left(\sum_{n=0}^{k}\left(u_{n+1}^{2}+u_{n}^{2}\right)\right)^{1 / 2}\right]^{p} \\
& =\alpha\left(c^{*}(k+1)\right)^{p} 2^{p / 2}\|u\|^{p} .
\end{aligned}
$$

So, by (30), we get

$$
\begin{gathered}
J(u)=\frac{1}{p} \sum_{n=-1}^{k} r_{n}\left|\Delta^{2} u_{n}\right|^{p}-\sum_{n=0}^{k} F\left(n, u_{n+1}, u_{n}\right) \\
\geq\left[\frac{r_{*}}{p}\left(c_{*}(k+2)\right)^{p}\left(\lambda_{*}(k+1) \lambda_{*}(k)\right)^{p / 2}\right. \\
\left.\quad-\alpha\left(c^{*}(k+1)\right)^{p} 2^{p / 2}\right]\|u\|^{p} \\
=\left(c^{*}(k+1)\right)^{p} 2^{p / 2}\left(\alpha_{0}-\alpha\right)\|u\|^{p} .
\end{gathered}
$$

Since $\alpha<\alpha_{0}$, we let $a=\left(c^{*}(k+1)\right)^{p} 2^{p / 2}\left(\alpha_{0}-\alpha\right) \delta^{p}$ and $\rho=\delta$. Then by (49),

$$
J(u) \geq a, \quad \forall u \in \partial B_{\rho}
$$

which means that $J$ satisfies the condition $\left(J_{1}\right)$ of the Mountain pass lemma.

By our assumptions, it is clear that $J(0)=0$. In order to use Mountain pass lemma, it suffices to verify that condition $\left(J_{2}\right)$ holds. In fact, similar to the proof of (45), we have

$$
J(u) \leq\left(c_{*}(k+1)\right)^{p} 2^{p / 2}\left(\beta_{0}-\beta\right)\|u\|^{p}+(k+1) \gamma,
$$

for any $u \in E$. Since $\beta_{0}<\beta$, it is easy to see that there exists an $e \in E$ with $\|e\|>\rho$ such that $J( \pm e)<0$. Thus $\left(J_{2}\right)$ holds.

According to Mountain pass lemma, $J$ possesses a critical value $c \geq a$ given by

$$
c=\inf _{g \in \Gamma_{1}} \max _{s \in[0,1]} J(g(s))
$$

where

$$
\Gamma_{1}=\{g \in C([0,1], E) \mid g(0)=0, g(1)=e\} .
$$

Let $\bar{u} \in E$ be a critical point of $J$ corresponding to the critical value $c$; then $\bar{u}$ is nontrivial and $J(\bar{u})=c$.

On the other hand, by (51), we have

$$
\lim _{\|u\| \rightarrow \infty} J(u)=-\infty
$$

Since $E$ is a $k$-dimensional space, by the continuity of $J(u)$ on $u$, we see that there exists $\widehat{u} \in E$ such that

$$
J(\widehat{u})=\max \{J(u) \mid u \in E\} .
$$

Clearly, $\widehat{u}$ is a nonzero critical point of $J$, and $J(\widehat{u}) \geq c \geq a>0$.

If $\widehat{u} \neq \bar{u}$, then the proof is finished. Otherwise, $\widehat{u}=\bar{u}$. Since $\|-e\|>\rho$ and $J(-e)<0$, then by Mountain pass lemma again, $J$ possesses a critical value $\tilde{c} \geq a$ given by

$$
\widetilde{c}=\inf _{g \in \Gamma_{2}} \max _{s \in[0,1]} J(g(s))
$$

where

$$
\Gamma_{2}=\{g \in C([0,1], E) \mid g(0)=0, g(1)=-e\} .
$$

Let $\tilde{u} \in E$ be a critical point of $J$ corresponding to the critical value $\widetilde{c}$. If $\widetilde{u} \neq \widehat{u}$, then the proof is finished. Otherwise $\tilde{u}=\widehat{u}=\bar{u}$. Let $g_{1}(s)=$ se for $s \in[0,1]$; then $g_{1} \in \Gamma_{1}$. By the definition of $c$, we see that there exists $s_{1} \in(0,1)$ such that $J\left(s_{1} e\right)=J(\widehat{u})$. Thus $s_{1} e$ is a critical point of $J$. Similar, let $g_{2}(s)=-s e$ for $s \in[0,1]$; then $g_{2} \in \Gamma_{2}$. By the definition of $\widetilde{c}$, we see that there exists $s_{2} \in(0,1)$ such that $J\left(-s_{2} e\right)=J(\widehat{u})$. And $-s_{2} e$ is a critical point of $J$. Clearly $s_{1} e \neq-s_{2} e$. The proof is now completed.

In the last part of this paper, we give an example to illustrate our results. 
Example 10. Consider (1) with (2), where $f$ is defined by

$$
\begin{aligned}
f(n, u, v, w) & \\
= & \frac{2 \mu_{n} v\left(u^{2}+v^{2}\right)^{p / 2}}{1+u^{2}+v^{2}} \\
& +\mu_{n} p v \ln \left(1+u^{2}+v^{2}\right)\left(u^{2}+v^{2}\right)^{(p / 2)-1} \\
& +\frac{2 \mu_{n-1} v\left(v^{2}+w^{2}\right)^{p / 2}}{1+v^{2}+w^{2}} \\
& +\mu_{n-1} p v \ln \left(1+v^{2}+w^{2}\right)\left(v^{2}+w^{2}\right)^{(p / 2)-1},
\end{aligned}
$$

for $n \in \mathbb{Z}(1, k)$. Here $\mu_{n}>0$ for $n \in \mathbb{Z}(0, k)$. Define

$$
\begin{array}{r}
F(n, u, v)=\mu_{n} \ln \left(1+u^{2}+v^{2}\right)\left(u^{2}+v^{2}\right)^{p / 2}, \\
n \in \mathbb{Z}(0, k) .
\end{array}
$$

Then $F(n, 0,0)=0$ for $n \in \mathbb{Z}(0, k)$ and (10) holds. Moreover, it is easy to see that $F(n, u, v)$ satisfies (39) for $n \in \mathbb{Z}(0, k)$. By Corollary 7, we see that (1) with (2) when $f$ is defined by (58) has at least two nontrivial solutions.

\section{Conflict of Interests}

The author declares that there is no conflict of interests regarding the publication of this paper.

\section{Acknowledgments}

The author would like to thank the anonymous referee for his/her valuable suggestions. This work is supported by Program for Changjiang Scholars and Innovative Research Team in University (no. IRT1226), the National Natural Science Foundation of China (no. 11171078), and the Specialized Fund for the Doctoral Program of Higher Education of China (no. 20104410110001).

\section{References}

[1] J. Henderson and H. B. Thompson, "Existence of multiple solutions for second-order discrete boundary value problems," Computers \& Mathematics with Applications, vol. 43, no. 10-11, pp. 1239-1248, 2002.

[2] Y. Li and J. Shu, "Solvability of boundary value problems with Riemann-Stieltjes $\Delta$-integral conditions for second-order dynamic equations on time scales at resonance," Advances in Difference Equations, vol. 42, pp. 8-18, 2011.

[3] R. Ma and C. Gao, "Bifurcation of positive solutions of a nonlinear discrete fourth-order boundary value problem," Zeitschrift für Angewandte Mathematik und Physik, vol. 64, no. 3, pp. 493506, 2013.

[4] C. Yuan, "Positive solutions of a singular positone and semipositone boundary value problems for fourth-order difference equations," Discrete Dynamics in Nature and Society, vol. 2010, Article ID 312864, 16 pages, 2010.

[5] L. Gao, "Existence of multiple solutions for a second-order difference equation with a parameter," Applied Mathematics and Computation, vol. 216, no. 5, pp. 1592-1598, 2010.
[6] H. Liang and P. Weng, "Existence and multiple solutions for a second-order difference boundary value problem via critical point theory," Journal of Mathematical Analysis and Applications, vol. 326, no. 1, pp. 511-520, 2007.

[7] X. Deng and H. Shi, "On boundary value problems for second order nonlinear functional difference equations," Acta Applicandae Mathematicae, vol. 110, no. 3, pp. 1277-1287, 2010.

[8] J. Liu, S. Wang, and J. Zhang, "Multiple solutions for boundary value problems of second-order difference equations with resonance," Journal of Mathematical Analysis and Applications, vol. 374, no. 1, pp. 187-196, 2011.

[9] Q. R. Zou and P. X. Weng, "Solutions of 2nth-order boundary value problem for difference equation via variational method," Advances in Difference Equations, vol. 2009, Article ID 730484, 10 pages, 2009.

[10] D. Bai and Y. Xu, "Nontrivial solutions of boundary value problems of second-order difference equations," Journal of Mathematical Analysis and Applications, vol. 326, no. 1, pp. 297302, 2007.

[11] R. Zhang, "Positive solutions of BVPs for third-order discrete nonlinear difference systems," Journal of Applied Mathematics and Computing, vol. 35, no. 1-2, pp. 551-575, 2011.

[12] J. Yu and Z. Guo, "Boundary value problems of discrete generalized Emden-Fowler equation," Science in China A, vol. 49, no. 10, pp. 1303-1314, 2006.

[13] T. He and Y. Xu, "Positive solutions for nonlinear discrete second-order boundary value problems with parameter dependence," Journal of Mathematical Analysis and Applications, vol. 379, no. 2, pp. 627-636, 2011.

[14] H. Berger, "Existence of nontrivial solutions of a two point boundary value problem for a $2 n$th order nonlinear difference equation," Advances in Dynamical Systems and Applications, vol. 3, no. 1, pp. 131-146, 2008.

[15] S. Xie and J. Zhu, "Positive solutions of the system for $n$ th-order singular nonlocal boundary value problems," Journal of Applied Mathematics and Computing, vol. 37, no. 1-2, pp. 119-132, 2011.

[16] S. Huang and Z. Zhou, "On the nonexistence and existence of solutions for a fourth-order discrete boundary value problem," Advances in Difference Equations, vol. 2009, Article ID 389624, 18 pages, 2009.

[17] R. P. Agarwal, Difference Equations and Inequalities: Theory, Methods, and Applications, vol. 228, Marcel Dekker, New York, NY, USA, 2000.

[18] H. Shi, Z. Liu, and Z. Wang, "Dirichlet boundary value problems for second order $p$-Laplacian difference equations," Rendiconti dell'Istituto di Matematica dell'Università di Trieste, vol. 42, pp. 19-29, 2010.

[19] X. Liu, Y. Zhang, B. Zheng, and H. Shi, "Periodic and subharmonic solutions for second order $p$-Laplacian difference equations," Indian Academy of Sciences, vol. 121, no. 4, pp. 457468, 2011.

[20] J. Mawhin, "Periodic solutions of second order nonlinear difference systems with $p$-Laplacian: a variational approach," Nonlinear Analysis: Theory, Methods \& Applications, vol. 75, no. 12, pp. 4672-4687, 2012.

[21] Z. Zhou, J. Yu, and Z. Guo, "Periodic solutions of higherdimensional discrete systems," Proceedings of the Royal Society of Edinburgh A, vol. 134, no. 5, pp. 1013-1022, 2004.

[22] P. H. Rabinowitz, Minimax Methods in Critical Point Theory with Applications to Differential Equations, vol. 65, American Mathematical Society, Providence, RI, USA, 1986. 


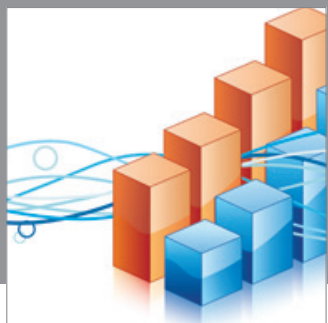

Advances in

Operations Research

mansans

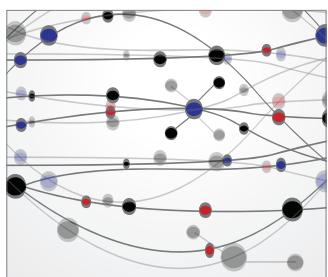

The Scientific World Journal
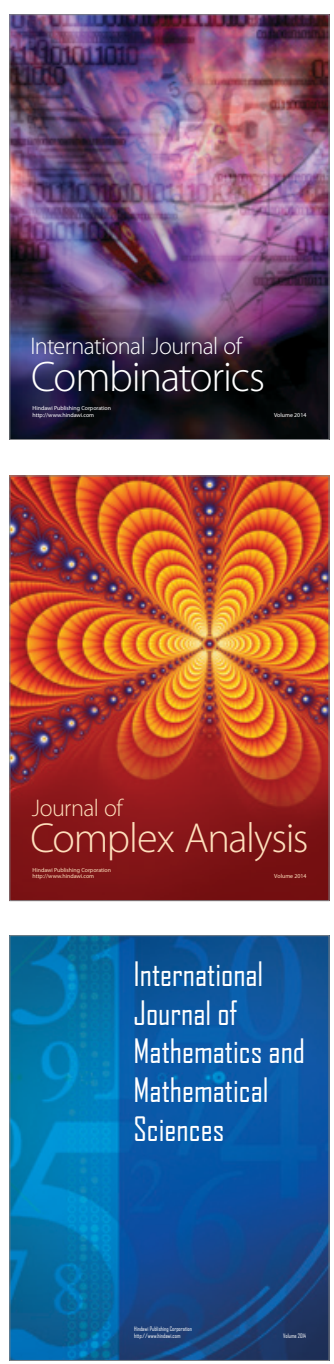
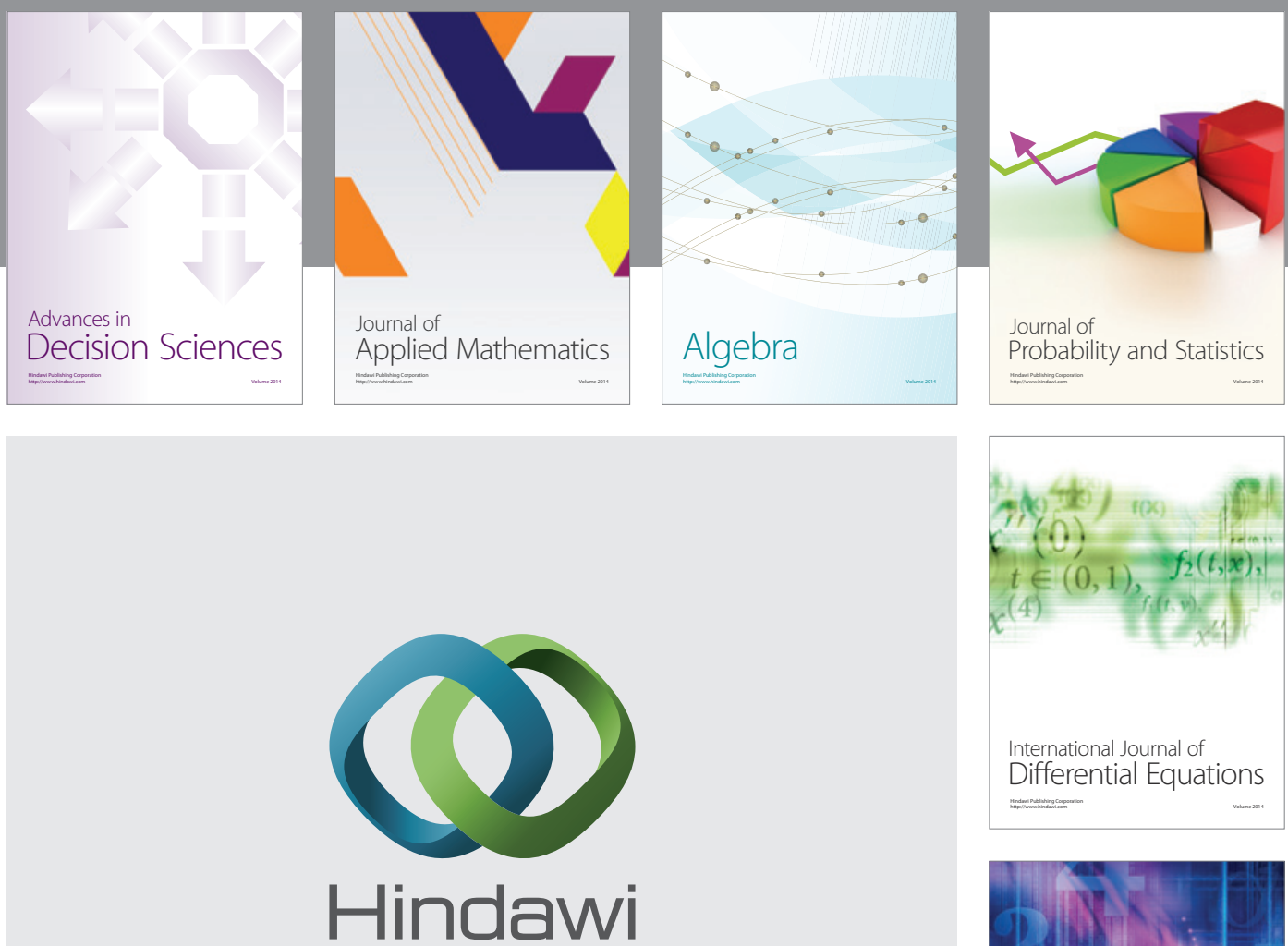

Submit your manuscripts at http://www.hindawi.com
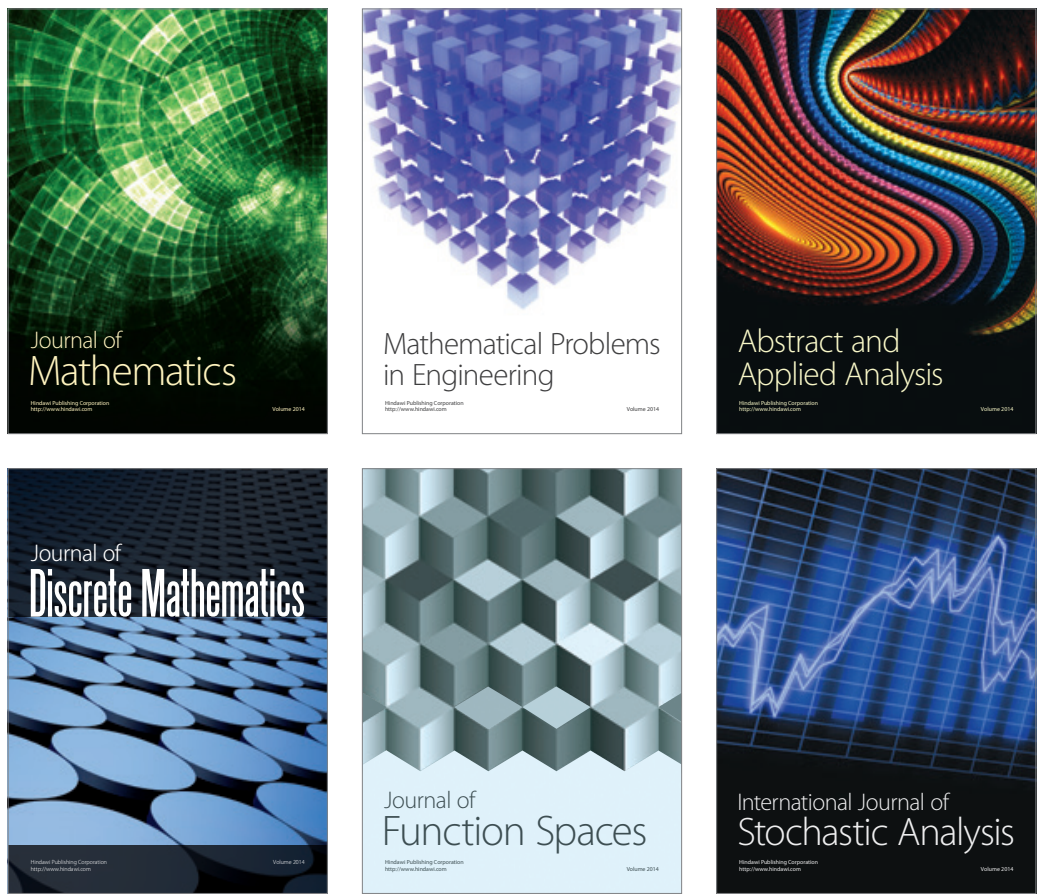

Journal of

Function Spaces

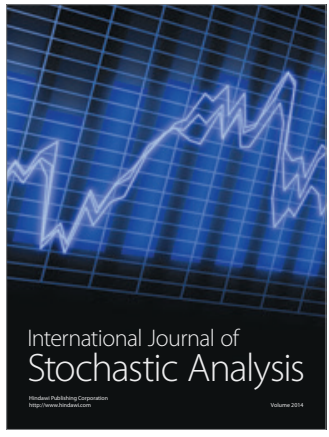

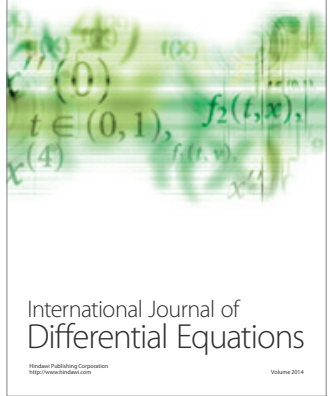
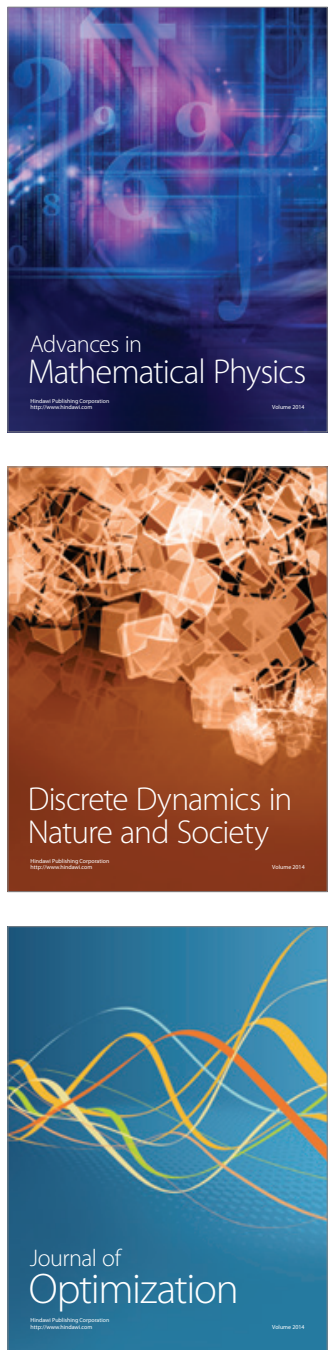